\title{
The Influence area of a subsonically expanding ellipsoidal inclusion
}

\author{
Xiaojun Su \& Surong Huang \\ Department of Aeronautics, Xiamen University, Fujian, China
}

ABSTRACT: The expression for the elastic field of an expanding ellipsoidal inclusion in an infinite isotropic elastic medium is presented. The influence area of a subsonically expanding ellipsoidal inclusion is given in the form of expressions and graphs. And the difference of the influence area between ellipsoidal inclusion and spherical inclusion under subsonically expanding is discussed.

KEYWORD: Influence area; Subsonic expansion; ellipsoidal; inclusion

\section{INTRODUCTION}

The elastic field of an infinite isotropic elastic medium is investigated when an ellipsoidal portion of the medium experiences a dynamic phase transformation. The phase transformation is nucleated from a point and expanding at a speed of time profile, where $\Omega$ (Fig.1)is given by

$x_{1}^{2} / a_{1}^{2}(t)+x_{2}^{2} / a_{2}^{2}(t)+x_{3}^{2} / a_{3}^{2}(t) \leq 1$

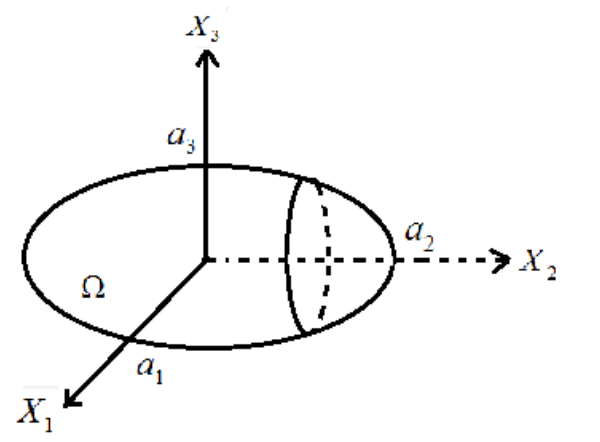

For convenience, we consider a suddenly transformed ellipsoidal inclusion and assume that the inclusion expands at a constant speed lower than wave speeds with a pure dilatational eigenstrain. Two special shapes as oblate spheroid and prolate spheroid will be discussed later.

Using the Fourier-transformation method and the 3-D elasto-dynamic Green's function, the displacement field according to paper[1] is given by:

$\mathrm{u}_{\mathrm{i}}(\mathrm{X}, \mathrm{t})=\int_{0}^{t} \iint_{s\left(t^{\prime}\right)} \mathrm{C}_{m n k} \varepsilon^{*} \varepsilon_{k l}^{*} f\left(t^{\prime}\right) n_{n}\left(X^{\prime}\right) u_{i m}^{\mathrm{G}}\left(\mathrm{X}-\mathrm{X}^{\prime}, t-t^{\prime}\right) d \mathrm{X}^{\prime} d t^{\prime}$,

Where $n_{n}\left(X^{\prime}\right)$ is the n-th component of outward normal to the inclusion surface $\mathrm{S}$, and $u_{i m}^{\mathrm{G}}(\mathrm{X}, t)$ is the three-dimensional elasto-dynamic Green's function with explicit form as follows:

Fig.1 The expanding ellipsoidal inclusion

$$
\mathrm{u}_{\mathrm{im}}^{\mathrm{G}}\left(\mathrm{X}-\mathrm{X}^{\prime}, t-t^{\prime}\right)=\left\{\begin{array}{l}
\frac{1}{4 \pi \rho}\left\{\left(t-t^{\prime}\right) \frac{\partial^{2}}{\partial x_{i} \partial x_{m}} \frac{1}{x}+\frac{1}{c_{2}} \delta\left[x-c_{2}\left(t-t^{\prime}\right)\right] \frac{\partial^{2}}{\partial x_{i} \partial x_{m}} x+\frac{\left(x_{i}-y_{i}\right)\left(x_{m}-y_{m}\right)}{c_{1} x^{3}} \delta\left[x-c_{1}\left(t-t^{\prime}\right)\right]\right\} \\
\text { for } \frac{x}{c_{1}} \leq t-t^{\prime} \leq \frac{x}{c_{2}} \\
0, \text { otherwise }
\end{array}\right.
$$

Where $c_{1}$ and $c_{2}$ are longitudinal wave speed and shear wave speed accordingly:

$$
\mathrm{c}_{1}=\sqrt{\frac{2 \mu+\lambda}{\rho}}, \mathrm{c}_{2}=\sqrt{\frac{\mu}{\rho}} .
$$




\section{INFLUENCE AREA}

To solve the integration in Eq.(2), we introduce the concept of influence areas(paper[1]). For some fixed space point $X$ (observation point), the influence area is defined as the collection of source points from which stress waves are emitted influencing the displacement of the observation point.

The influence areas at time $t$ with respect to the longitudinal wave and shear wave are defined as:

For longitudinal wave

$$
\mathrm{A}_{\alpha}=\left\{\mathrm{X}^{\prime} \| \mathrm{X}^{\prime}|\leq \Omega(t),| \mathrm{X}-\mathrm{X}^{\prime} \mid \leq c_{1}\left(t-t^{\prime}\right), 0 \leq t^{\prime} \leq t\right\}
$$

For shear wave

$$
\mathrm{A}_{\beta}=\left\{\mathrm{X}^{\prime}|| \mathrm{X}^{\prime}|\leq \Omega(t),| \mathrm{X}-\mathrm{X}^{\prime} \mid \leq c_{2}\left(t-t^{\prime}\right), 0 \leq t^{\prime} \leq t\right\}
$$

Here $X^{\prime}$ indicate the points on boundary of $\Omega\left(t^{\prime}\right)$.

The shape and size of the influence area is influenced by the position of the observation point $\mathrm{X}$ and time t. The integration in Eq. (2) then should be performed on the influence areas $\mathrm{A}_{\alpha}-\mathrm{A}_{\beta}$. To simplify the problem, the observation point is set on the $x_{3}$-axis with coordinates $(0,0, \mathrm{~L})$. As mentioned before, two special shapes as oblate spheroid and prolate spheroid will be discussed below.

\section{INFLUENCE AREA OF OBLATE SPHEROID}

Here, the elliptical shape factors are given as:

$$
\mathrm{a}_{1}(\mathrm{t})=\mathrm{a}_{2}(\mathrm{t})>\mathrm{a}_{3}(\mathrm{t}),
$$

According to the arrival sequence of the longitudinal wave and shear wave as well as whether the observation point is included into the inclusion or not, the following cases are discussed (Fig.2), somewhat alike to the figure of influence area of an expanding spherical inclusion in paper[1]:
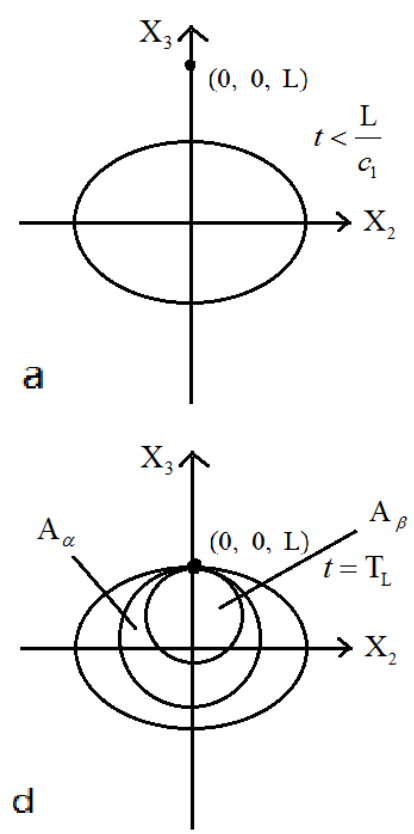

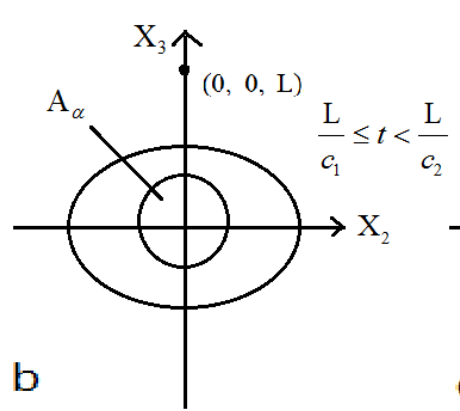

C
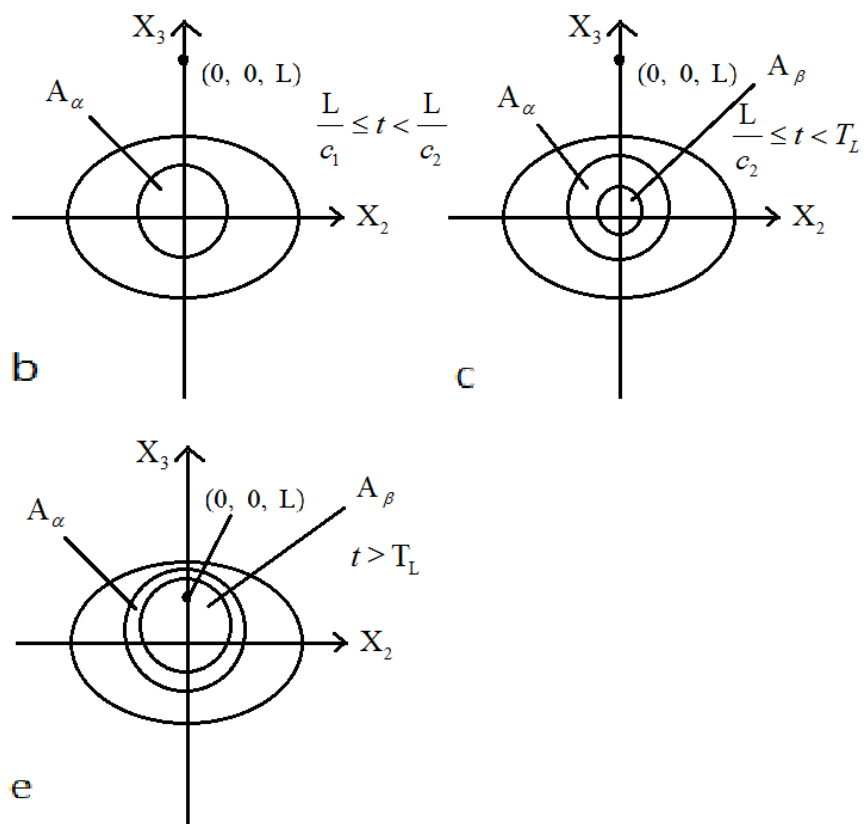

Fig. 2 The influence area of a subsonically expanding oblate spheroid inclusion at different time stage

$$
\text { (a) } t<\frac{\mathrm{L}}{c_{1}} \text {, }
$$

This is the case that both waves have not arrived at the observation point, which means neither $\mathrm{A}_{\alpha}$ nor $\mathrm{A}_{\beta}$ arise, as shown in Fig.2a.

(b) $\frac{\mathrm{L}}{C_{1}} \leq t<\frac{\mathrm{L}}{\mathrm{C}_{2}}$,

This is the case that some of the longitudinal waves have arrived at the observation point, but the shear waves have not. The absolute value of the intersection of $\mathrm{A}_{\alpha}$ with the $\mathrm{x}_{3}$ axis are denoted as $a_{31}=a_{3}\left(t_{1}\right), a_{32}=a_{3}\left(t_{2}\right)$, with the $\mathrm{x}_{2}$ axis denoted as $a_{23}=a_{2}\left(t_{3}\right), a_{24}=a_{2}\left(t_{4}\right)$, as shown in Fig.2b. It is easy to show that $a_{31}, a_{32} a_{23}$ and $a_{24}$ satisfy the following equations:

$$
\begin{aligned}
& \left(t-t_{1}\right) c_{1}=\mathrm{L}+a_{31}=\mathrm{L}+a_{3}\left(t_{1}\right), \\
& \left(t-t_{2}\right) c_{1}=\mathrm{L}-a_{32}=\mathrm{L}-a_{3}\left(t_{2}\right), \\
& \left(t-t_{3}\right) c_{1}=\sqrt{\mathrm{L}^{2}+a_{23}^{2}}=\sqrt{\mathrm{L}^{2}+a_{2}^{2}\left(t_{3}\right),}
\end{aligned}
$$


$\left(t-t_{4}\right) c_{1}=\sqrt{\mathrm{L}^{2}+a_{24}^{2}}=\sqrt{\mathrm{L}^{2}+a_{2}^{2}\left(t_{4}\right)}$.

We can easily learn that $a_{31}<a_{32}$, but we cannot draw a certain conclusion whether $a_{31}<a_{23}$, it depends on $t, t_{1}$ and $t_{3}$. Only when the equation $\left(t-t_{1}\right) c_{1}-L=\sqrt{\left(t-t_{3}\right)^{2} c_{1}^{2}-L^{2}} \quad$ is satisfied, $a_{31}$ equals to $a_{32}$.

(c) $\frac{\mathrm{L}}{c_{2}} \leq t<T_{L},\left(a_{3}\left(T_{L}\right)=L\right)$,

At that time, $\mathrm{A}_{\beta}$ comes into being. Accordingly, the absolute value of the intersection of $\mathrm{A}_{\beta}$ with the $\mathrm{x}_{3}, \mathrm{x}_{2}$ axis are denoted as $a^{\prime}{ }_{31}=a_{3}{ }^{\prime}\left(t_{1}{ }^{\prime}\right)$, $a_{32}^{\prime}=a_{3}{ }^{\prime}\left(t_{2}{ }^{\prime}\right), a_{23}^{\prime}=a_{2}{ }^{\prime}\left(t_{3}{ }^{\prime}\right)$ and $a^{\prime}{ }_{24}=a_{2}{ }^{\prime}\left(t_{4}{ }^{\prime}\right)$. The equations which $a_{31}^{\prime}, a_{32}^{\prime} a_{23}^{\prime}$ and $a_{24}^{\prime}$ satisfy have the similar form as equations (8.1)-(8.4) for $\mathrm{A}_{\alpha}$.

\section{(d) $t \geq \mathrm{T}_{\mathrm{L}}$.}

As shown in Fig.2d and e, the observation point is now included inside the inclusion. Particularly when $t=\mathrm{T}_{\mathrm{L}}$, both $a_{32}$ and $a_{32}^{\prime}$ are equal to $L$. After that, the equations for $a_{32}$ and $a_{32}^{\prime}$ change to :

$\left(t-t_{2}\right) c_{1}=a_{32}-L=a_{3}\left(t_{2}\right)-L$,

$\left(t-t_{2}^{\prime}\right) c_{1}=a_{32}^{\prime}-L=a_{3}\left(t_{2}^{\prime}\right)-L$.

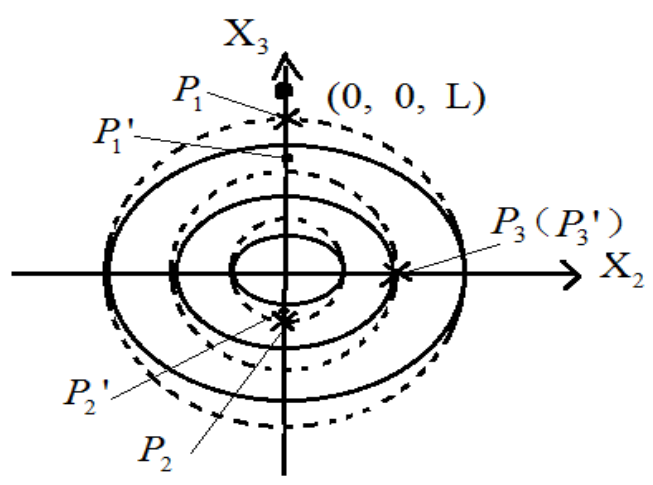

a compare sphercial case with oblate case

\section{INFLUENCE AREA OF PROLATE SPHEROID}

Here, the elliptical shape factor are given as:

$\mathrm{a}_{1}(\mathrm{t})=\mathrm{a}_{2}(\mathrm{t})<\mathrm{a}_{3}(\mathrm{t})$

The influence area of an expanding prolate spheroid is similar to the one of an expanding oblate spheroid in relative location with the inclusion while much narrower in shape.

\section{COMPARING WITH SPHERICAL CASE}

In order to make clear the difference between the influence areas of spheroid and spherical case in paper[1], some specific point pairs both on the boundary of $\mathrm{A}_{\alpha}$ at a specific time $T$ but different in shape of inclusion are made comparison. The radius of an expanding sphere is defined as $\mathrm{R}(\mathrm{t})$.

As shown in Fig.3, we assume that $\mathrm{a}_{1}(\mathrm{t})=\mathrm{a}_{2}(\mathrm{t})=\mathrm{R}(\mathrm{t})$, the dashed represents sphere inclusion at $t_{1}{ }^{\prime}, t_{2}{ }^{\prime}$ and $t_{3}{ }^{\prime}$, and the $\mathrm{X}$ marks $P_{1}, P_{2}, P_{3}$ indicate points on the boundary of $\mathrm{A}_{\alpha}$ at time $T$, while the full line represents spheroid and the spots indicate the corresponding point of spheroid case. The spherical and spheroid inclusion which overlap on $X_{2}$ axis are expanding at the same time of $t_{\mathrm{n}}{ }^{\prime}$.

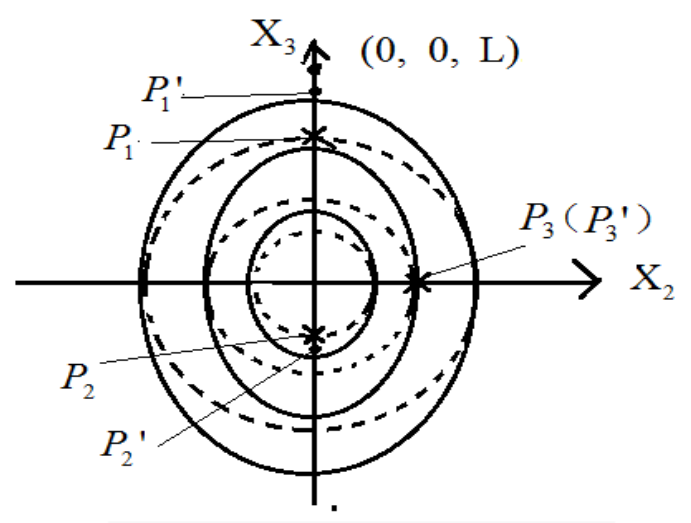

b compare sphercial case with prolate case

Fig.3 Specific points on the boundary of $\mathrm{A}_{\alpha}$ on spherical and spheroid inclusion

In Fig.3a, $P_{1}$ is given as the intersection of the $X_{3}$ axis with the surface of $\mathrm{R}\left(\mathrm{t}_{1}{ }^{\prime}\right)$, also on the boundary of $\mathrm{A}_{\alpha}$. But the intersection of the $X_{3}$ axis with the surface of $\Omega\left(\mathrm{t}_{1}^{\prime}\right)$ in oblate case is no longer included in $\mathrm{A}_{\alpha}$. In fact, the point $P_{1}{ }^{\prime}$ which somewhat lower in figure and earlier at emitted time t' could be on the boundary of $\mathrm{A}_{\alpha}$ at time $T$. Under this train of thought, when a sphere inclusion changes into an oblate spheroid, $P_{2}$ changes into $P_{2}{ }^{\prime}$, which also below the intersection on surface of $\Omega\left(t_{2}{ }^{\prime}\right)$. Besides, $P_{3}$ and $P_{3}$ ' overlap due to locating on the $X_{2}$ axis. We can learn that the influence area of an ex- 
panding oblate spheroid has a trend of far away from the observation point except the points on $X_{2}$ axis, compared to the influence area of an expanding sphere.

As for prolate case, the opposite trend is found, as shown in Fig.3b.

\section{CONCLUSION}

The elastic field of an expanding ellipsoidal inclusion in an infinite isotropic elastic medium is presented with Green's function method. The influence area of the ellipsoidal inclusion expanding in subsonic speed is discussed and compared with that of spherical case.

\section{ACKNOWLEDGEMENTS}

This work was financially supported by the National Natural Science Foundation of China (Grant No.11102174) and the Scientific Research Foundation for the Returned Overseas Chinese Scholars, State Education Ministry of China.

\section{REFERENCES}

[1] Z.M.Xiao and J.Luo: On the transient elastic field for an expanding spherical inclusion in 3-D solid. Acta Mechanica.163, 147-159(2003). 